\title{
Signs and Symbols
}

[] enclose phonetic transcription

// enclose phonemic transcription

<> enclose graphemes

$>$ yields

$\rightarrow$ indicates semantic or derivational changes

$>\rightarrow$ indicates morphological/analogidaadjustmentsin addition to direct sound change

* reconstructed

$x$ indicates number of occurrences of a certain word/feature

$\sim$ varies with

:: indicates that two terms/expressions are parts of the same morphological system

- indicates the second member of a compound 
